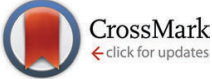

Cite this: Phys. Chem. Chem. Phys., 2015, 17, 26463

Received 29th June 2015,

Accepted 8th September 2015

DOI: $10.1039 / c 5 c p 03751 d$

www.rsc.org/pccp

\section{Six-fold-symmetry internal rotation in toluenes: the low barrier challenge of 2,6- and 3,5-difluorotoluene $\dagger$}

\author{
K. P. Rajappan Nair, ${ }^{a}$ Michaela K. Jahn, ${ }^{* a}$ Alberto Lesarri, ${ }^{b}$ Vadim V. Ilyushin ${ }^{c}$ and \\ Jens-Uwe Grabow ${ }^{a}$
}

\begin{abstract}
Pure six-fold symmetry $\left(V_{6}\right)$ internal rotation poses significant challenges to experimental and theoretical determination, as the very low torsional barriers result in huge tunneling splittings difficult to identify and to model. Here we resolved the methyl group internal rotation dynamics of 2,6- and 3,5-difluorotoluene using a newly developed computer code especially adapted to $V_{6}$ problems. The jet-cooled rotational spectra of the title molecules in the $5-25 \mathrm{GHz}$ region revealed internal rotation tunneling doublings of up to $3.6 \mathrm{GHz}$, which translated in methyl group potential barriers of $V_{6}=0.14872(24)$ and $0.0856(10) \mathrm{kJ} \mathrm{mol}^{-1}$, respectively, in the vibrational ground-state. Additional information on Stark effects and carbon isotopic species in natural abundance provided structural data and the electric dipole moments for both molecules. $A b$ initio calculations at the MP2 level do not reproduce the tiny torsional barriers, calling for experiments on other systems and additional theoretical models.
\end{abstract}

\section{Introduction}

The study of conformational problems arising from hindered internal motions is a classic topic in structural Chemistry. ${ }^{1}$ Even for small molecules the internal rotation around single bonds may result in a large number of rotamers, and a detailed description of the conformational distribution requires investigating both the spectral signature and the potential energy surface of the molecule. Among the experimental methods the investigation of the pure rotational spectrum in the microwave region provides the most accurate description of the molecular structure, ${ }^{2,3}$ since the sensitivity of the moments of inertia to the mass distribution results in independent spectra for all species in the sample, either rotamers or tautomers. For terminal methyl groups the internal rotation effects produce tunneling splittings in the rotational transitions, from which the barrier potentials can be precisely determined. ${ }^{4,5}$ This method has been applied

\footnotetext{
${ }^{a}$ Institut für Physikalische Chemie und Elektrochemie, Gottfried Wilhelm Leibniz Universität Hannover, Callinstraße 3A, 30167 Hannover, Germany.

E-mail: Michaela.jahn@pci.uni-hannover.de; Fax: +49 (511)762-4009; Tel: $+49(511) 762-5278$

${ }^{b}$ Departamento de Quimica Física y Quimica Inorgánica, Facultad de Ciencias, Universidad de Valladolid, 47011 Valladolid, Spain

${ }^{c}$ Institute of Radio Astronomy of NASU, Chervonopraporna 4, 61002 Kharkov, Ukraine

$\dagger$ Electronic supplementary information (ESI) available: Supplementary data associated with this article can be found in the online version. See DOI: $10.1039 / \mathrm{c} 5 \mathrm{cp} 03751 \mathrm{~d}$
}

extensively for many three-fold $V_{3}$ barriers, which occur when the methyl top is attached to a molecular frame of $C_{1}$ or $C_{\mathrm{s}}$ symmetry. Higher terms in the $(\cos 3 n \tau)$ torsion potential expansion, in particular $V_{6}$, are usually small in comparison with the leading $V_{3}$ term. However, for molecular frames of higher symmetry, like $C_{2 \mathrm{v}}$ aromatic rings, the internal rotation of a $C_{3 \mathrm{v}}$-symmetric methyl group around the $C_{2 \mathrm{v}}$ axis will be hindered by a leading six-fold $V_{6}$ potential term, giving an opportunity to examine the molecular properties governing this kind of barrier, much less studied in the literature. We recently started a systematic investigation of fluorinated toluenes and we report here our results for the internal rotation potentials determined from the microwave spectra of 2,6- and 3,5-difluorotoluene. While the toluenes are rigid backbone molecular systems for which the detection of the rotational spectrum could be relatively easy, the presence of low internal rotation barriers produces large (on the order of $\mathrm{GHz}$ ) frequency splittings, making the spectral assignment and analysis a considerable challenge. In the case of 2,6-difluorotoluene, many lines have splittings for the transitions in the lowest torsional states, i.e., $m=0$ and $m=1$, as large as $3 \mathrm{GHz}$ (e.g. $3.16 \mathrm{GHz}, 3.20 \mathrm{GHz}$ or $3.58 \mathrm{GHz}$ in the $6_{1,5} \leftarrow 5_{2,4}, 7_{3,5} \leftarrow 6_{2,4}$ or $8_{3,6} \leftarrow 7_{2,5}$ transitions, respectively).

The molecular prototype of six-fold internal rotation barriers is toluene. The initial measurements by Rudolph, Dreizler, Jaeschke and Wendling, ${ }^{6}$ restricted to cm-wave low- $J$ rigid-rotor transitions in the ground $(m=0)$ and $m=3$ free-rotor vibrational quantum states, led to a barrier of 13.94(10) $\mathrm{cal} \mathrm{mol}^{-1}\left(4.88(3) \mathrm{cm}^{-1}\right)$. Kisiel et al. extended the measurements first to the high 
$J m=0$ torsional ground state ${ }^{7}$ and then to a simultaneous analysis of $m=0,1,2,3$ and -3 states, ${ }^{8}$ finally leading to a potential barrier of $V_{6}=13.832068(3) \mathrm{cal} \mathrm{mol}^{-1}\left(4.8378362(9) \mathrm{cm}^{-1}\right)$. The rotational spectra of the toluene ${ }^{13} \mathrm{C}$ isotopologues are also known and are used for the structural determination. ${ }^{9,10}$ Recently Gascooke et al. ${ }^{11,12}$ reported an analysis of the torsion-vibration coupling mechanism in toluene that leads to a significant revision of the torsional barrier height in this molecule. This study raises an interesting question of interpretation of the results obtained for many molecules with large amplitude motions (LAMs) in the framework of widely used models which assume a possibility of separating a large amplitude vibrational motion from small amplitude vibrational motions in a nonrigid molecule at least for some selection of low lying states associated with LAMs. Whereas we believe that the barrier height revision of Gascooke et al. ${ }^{11,12}$ should be confirmed by further analysis of torsion-vibration coupling in toluene with incorporation of high-precision microwave data it is clear that investigation of similar molecular systems will be of great interest in resolving this controversy between different barrier height results. 2,6-difluorotoluene and 3,5-difluorotoluene seem to be good testing cases since at least for 2,6-difluorotoluene analogous torsion-vibration coupling was reported for the S1 state by Walker et $a l^{13}$ and therefore it is likely that the same coupling will be present in the S0 state also. Since jet cooled rotational spectra provide information only on low lying torsion-rotation states an analysis of possible vibration-torsion couplings in 2,6-difluorotoluene and 3,5-difluorotoluene was beyond the scope of our current investigation. At the same time our study provides a necessary basis for further investigation of higher excited torsional-vibrational states of these molecules using high-precision microwave spectroscopic techniques.

How will the six-fold torsional barrier of toluene be affected by $C_{2 \mathrm{v}}$-preserving halogen substitution in different positions of the ring? We found in the bibliography several rotational studies of monohalogenated toluenes ${ }^{14-18}$ but only a few of disubstituted ones, ${ }^{19}$ none with a six-fold barrier. For this reason we investigated several difluoro and dichloro derivatives. In this report we examine the results of symmetrically attaching two fluorine atoms $\left(\mathrm{C}_{7} \mathrm{H}_{6} \mathrm{~F}_{2}\right)$ in ortho- and meta-positions. Since fluorine produces no hyperfine structure due to nuclear quadrupole coupling we could concentrate on resolving the fine structure due to the internal rotation. The experimental study has been carried out using Fourier-transform
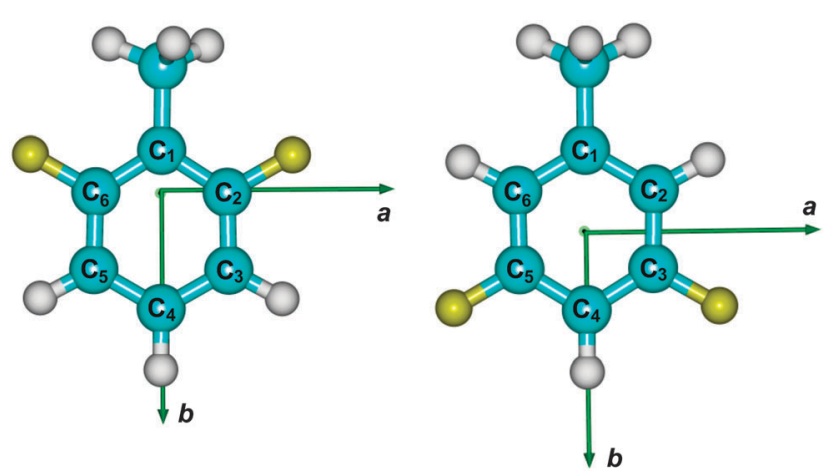

Fig. 1 Atom labelling and principal inertial axes of 2,6- and 3,5-difluorotoluene. microwave (FT-MW) spectroscopy in a supersonic jet. ${ }^{2}$ The adiabatic expansion not only reduces the collisional linewidth, but also produces a strong rovibrational cooling, populating only the lowest-lying states and thus simplifying the spectrum. The rotational analysis was supplemented by the determination of the molecular electric dipole moments using the Stark effect. Finally, we conducted $a b$ initio calculations on the two molecules to test the predictive value of the theoretical data against the experiment. Atom labelling and principal axis orientation in these molecules are shown in Fig. 1.

\section{Experimental and computational methods}

The spectra were taken on a pulsed supersonic jet Fourier transform microwave spectrometer in Hannover, using the coaxially oriented beam and resonator arrangement (COBRA) ${ }^{20-22}$ described before. The principal advantage in the COBRA arrangement is the longer transit time of the molecular jet, which can be exploited for Stark effect experiments if, rather than using external electrode plates, the spherical reflectors forming the resonator are utilized as high-voltage electrodes (coaxially aligned electrodes for Stark effect applied in resonators, $\mathrm{CAESAR}^{23}$ ). In the experiment the direction of the electric Stark field is perpendicular to the polarized microwave field, leading exclusively to the $\Delta M_{J}= \pm 1$ transitions. For the Stark effect measurements the applied voltages were calibrated for the electric field strengths using the Stark effect in the $J=1 \leftarrow 0$ rotational transition of the $\mathrm{OC}^{36} \mathrm{~S}$ and ${ }^{18} \mathrm{OCS}$ isotopologues of OCS using a dipole moment value of $\mu=0.71519(3) \mathrm{D},{ }^{24}$ as described in ref. 25. The substances, commercial samples of 2,6- and 3,5-difluorotoluene obtained from Alfa-Aesar $\mathrm{GmbH}$, were used without further purification. The sample was kept in a small reservoir at the nozzle exit, using neon as a carrier gas (a stagnation pressure of $200 \mathrm{kPa}$ ). The spectra were measured in the frequency range 5-25 GHz. All frequency measurements were referenced to a GPS-disciplined rubidium frequency standard and have an estimated uncertainty of $<1 \mathrm{kHz}$. Lines separated more than $\sim 5 \mathrm{kHz}$ are resolved.

$A b$ initio calculations were used to obtain predictions of the rotational parameters (centrifugal distortion and rotational constants), electric dipole moments and the torsional potential function. In the course of this work we used several computational models. We present in Table 1 the results of secondorder perturbation theory (MP2, frozen core), combined with a standard Pople's 6-311++G(2df,2p) basis set. The centrifugal distortion constants resulted from the harmonic force field. The calculations were performed with the GAUSSIAN package. ${ }^{26}$ The torsional potentials for the two molecules are shown in Fig. S1 (ESI $\dagger)$.

\section{Results}

\subsection{Rotational spectrum and the internal rotation barrier}

The first spectral predictions used rotational constants derived from the $a b$ initio methods. A large number of lines were then initially observed in the frequency region $10-16 \mathrm{GHz}$ 
Table 1 Rotational parameters of 2,6- and 3,5-difluorotoluene

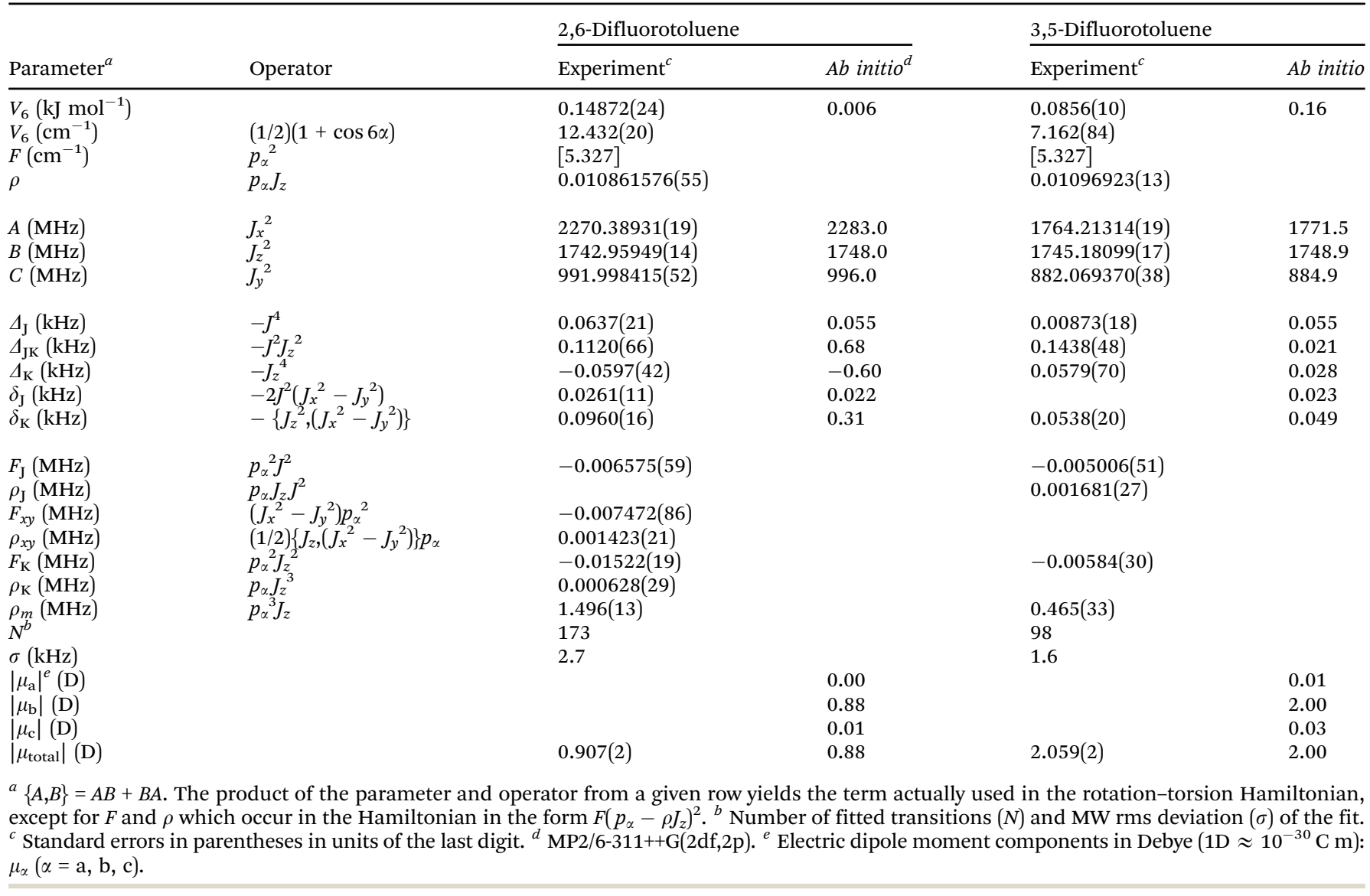

(Fig. 2 and Fig. S2, ESI $\dagger$ ). However, in order to rationalize the spectrum some considerations should be given on the torsional fine structure. The internal rotation governed by a leading $V_{6}$ barrier term splits the six-fold degeneracy of the ground vibrational state $(v=0)$ in a quartet of torsional vibrational states which can be labeled using free-rotor quantum numbers $m=0$, 1, 2, 3 ( $m=1$ and $m=2$ states are doubly degenerate). The torsion-rotation levels belonging to these $m$-states may be labeled according to the permutation-inversion molecular symmetry (MS) group $G_{12}$ i.e. $A_{1} / A_{2}, B_{1} / B_{2}, E_{1}, E_{2} \cdot{ }^{27}$ Spin statistics for 2,6- and 3,5-difluorotoluene, both having a $C_{3 \mathrm{v}}$-symmetric methyl group as well as pairs of equivalent protons and fluorine atoms if exchanged by $G_{12}$ symmetry operations, are 80, 48, 48 and 80 , respectively. Symmetry species exhibiting the same spin statistical weights were expected to relax to the lowest energy species within the stack. Under the experimental conditions of a supersonic jet (estimated effective rotational temperature of several $\mathrm{K}$ ) it was anticipated that only the $m=0$ and $m=1$ sub states of the ground vibrational state would be populated and each rotational transition due to internal rotation would appear as a doublet. Out of the two lines belonging to a particular rotational transition, the $m=0$ lines usually (in the absence of intertorsional interactions) effectively follow an asymmetric top pattern and hence the observed lines which were fitted by a rigid rotor asymmetric top model were identified as $m=0$ lines.
As there were no earlier predictions or determination of the potential barrier for 2,6- and 3,5-difluorotoluene from any other experimental method, we first estimated the splitting between the $m=0$ and $m=1$ lines with different assumed potential barriers obtained from chemical intuition and information from similar molecules. First we tried to map the $(m=0)-(m=1)$ splittings on the $A-E$ splittings of the usual three-fold problem using the XIAM program written by Hartwig, which employs the Combined Axis Method. ${ }^{28}$ This idea worked rather well, giving us an opportunity after several trial calculations to make initial assignments of a number of $m=1$ R-type transitions. Nevertheless rather high residuals for the fitted $m=1$ transition frequencies with respect to estimated experimental uncertainty as well as unexpectedly high values of "effective" barriers spoke in favor of using a code written specifically for the $V_{6}$ case.

The final analysis was based on the theoretical framework developed by Sorensen and Pedersen in their application of the Longuet-Higgins permutation-inversion group ideas to the microwave spectrum of $\mathrm{CH}_{3} \mathrm{NO}_{2} \cdot{ }^{29}$ We employed the program RAM36 (Rho-Axis-Method for 3- and 6- fold barriers), which uses the Rho Axis Method and was successfully applied previously to the analysis of the rotational spectra of both a light-top (toluene ${ }^{8}$ ) and a heavy-top molecule (benzotrifluoride ${ }^{30}$ ). A detailed description of the program can be found in those references. The computer code uses a least-squares optimizer that fits the values of selected Hamiltonian parameters to the set of observed 

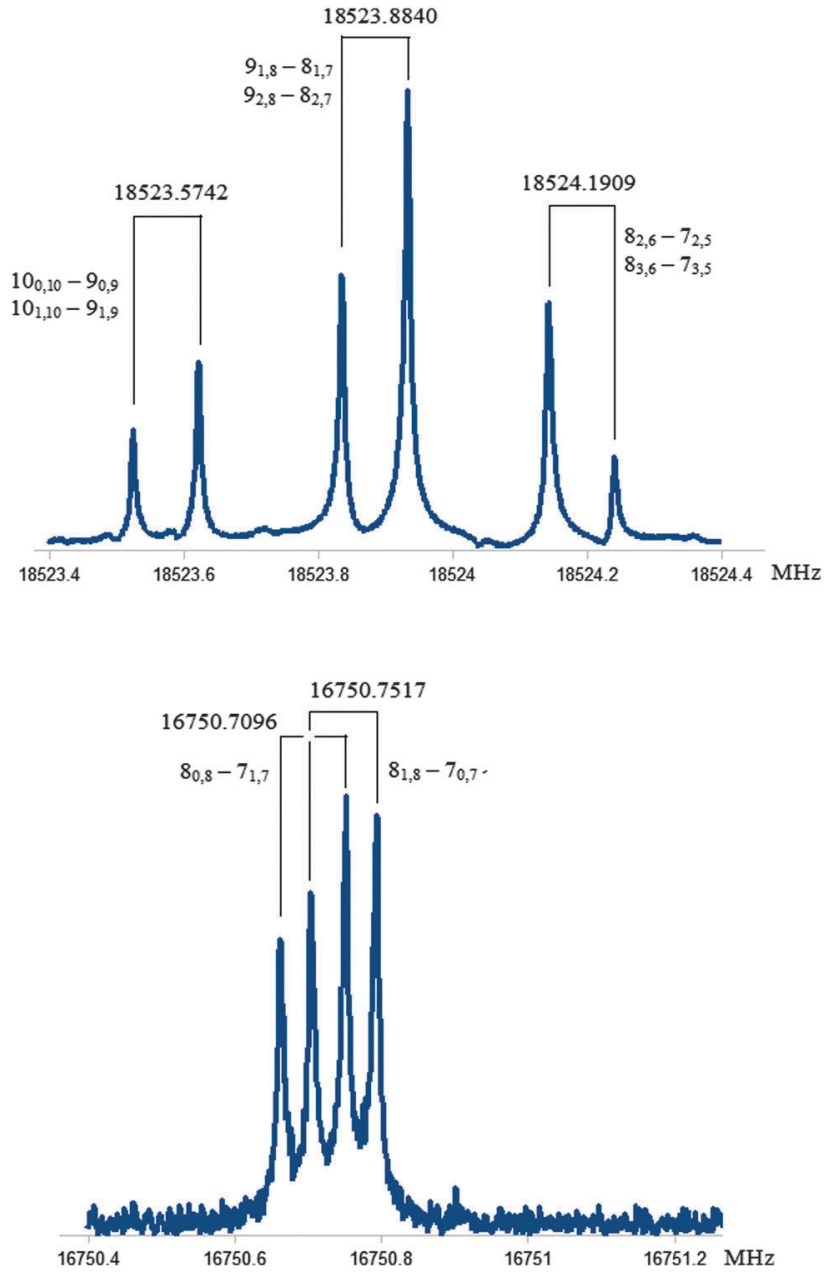

Fig. 2 An illustration of rotational transitions of 3,5-difluorotoluene (top trace, $m=0$ torsional state) and ${ }^{13} \mathrm{C}$ transitions of 2,6-difluorotoluene in natural abundance $\left({ }^{13} \mathrm{C}(3)\right.$ and ${ }^{13} \mathrm{C}(5)$, lower trace). All lines are split by the Doppler effect (the intensity has been normalized for clarity).

transition frequencies. It has a "global" nature in the sense that the whole set of energy levels associated with torsional motion of a $C_{3 \mathrm{v}}$ top is treated simultaneously with one set of parameters. $^{8}$

Although the 2,6- and 3,5-difluorotoluenes are derivatives of the toluene molecule, they are near-oblate tops in contrast to toluene (a prolate top with $\kappa=-0.59$ ). In these two molecules it is the $b$ axis which coincides with the methyl top internal rotation axis rather than the $a$ axis as in toluene. Therefore in our calculations we used the representation $\mathrm{II}^{1}(x, y, z=a, c, b)$. The Hamiltonian may be presented as follows:

$$
H=H_{\mathrm{r}}+H_{\mathrm{cd}}+H_{\mathrm{t}}+H_{\mathrm{tr}},
$$

with

$$
H_{\mathrm{t}}+H_{\mathrm{r}}=F\left(p_{\alpha}-\rho J_{z}\right)^{2}+(1 / 2) V_{6}(1+\cos 6 \alpha)+B J_{z}^{2}+A J_{x}^{2}+C J_{y}^{2}
$$

where $p_{\alpha}$ is an angular momentum conjugated to the internal rotation angle $\alpha$.

$H_{\mathrm{cd}}=-\Delta_{\mathrm{J}} \mathrm{P}^{4}-\Delta_{\mathrm{JK}} P^{2} P_{z}^{2}-\Delta_{\mathrm{K}} P_{z}^{4}-2 \delta_{\mathrm{J}} P^{2}\left(P_{x}^{2}-P_{y}^{2}\right)-\delta_{\mathrm{K}}\left\{P_{z}^{2},\left(P_{x}^{2}-P_{y}^{2}\right)\right\}$
The $H_{\text {tr }}$ contains higher order torsion-rotation interaction terms. In Table 1 only those terms which were actually used in the fits of the investigated spectra may be found.

The initial sets of R-type transitions assigned with the help of the XIAM program were refitted using the RAM36 program without much problem, giving rather good root-mean-square (rms) deviations of the fits for both $m=0$ and $m=1$ states of 2,6and 3,5-difluorotoluene. Based on this analysis we additionally assigned a number of $Q$-type transitions that gave us an opportunity to confirm the consistency of our assignments with the help of combination differences. As a final step we fulfilled a search for the rather weak $m=2$ lines, which according to our new predictions were at the detection limit of the FTMW spectrometer in use. We were able to assign $9 m=2$ lines for 2,6-difluorotoluene and only $3 \mathrm{~m}=2$ for 3,5-difluorotoluene using reasonable integration times.

In total, for 2,6-difluorotoluene we have fit 173 line frequencies that due to blending correspond to 176 transitions with angular momentum quantum numbers from $J=2$ up to $J=12$, among which assignment of 81 transitions was confirmed by combination differences. The theoretical model included 17 parameters: 5 parameters of the 2 nd order (one fixed) and 12 parameters of the 4 th order. The rms deviation of the fit was $2.7 \mathrm{kHz}$ with separate rms deviations for the $m=0,1,2$ states of $2.3 \mathrm{kHz}, 2.8 \mathrm{kHz}$ and $4.5 \mathrm{kHz}$. Although the obtained rms deviation is slightly higher than the estimated measurement uncertainty, it should be noted that a number of transitions (both in 2,6- and 3,5-difluorotoluene) were split presumably by spin-spin or spin-rotation interactions and therefore their frequency determination errors may be larger than the stated $1 \mathrm{kHz}$. To take this fact into account we weighted all measurements with uncertainties of $2 \mathrm{kHz}$ and in several cases $10 \mathrm{kHz}$ (the weight was proportional to the reciprocal of the squared uncertainty). The parameters resulting from the fit for 2,6-difluorotoluene are presented in Table 1.

For 3,5-difluorotoluene, in total, we have fit 98 line frequencies that due to blending correspond to 156 transitions with angular momentum quantum numbers $J=2$ up to $J=15$, among which the assignment of 46 transitions was confirmed by combination differences. The theoretical model included 14 parameters: 5 parameters of the 2 nd order (one fixed) and 9 parameters of the 4 th order. The rms deviation of the fit was $1.6 \mathrm{kHz}$ with separate rms deviations for $m=0,1,2$ states being $1.1 \mathrm{kHz}, 1.9 \mathrm{kHz}$ and $1.2 \mathrm{kHz}$. The resulting parameters for 3,5-difluorotoluene are also shown in Table 1.

It should be noted that besides the fact that we were able to measure several $m=2$ lines the torsion parameters in both 2,6- and 3,5-difluorotoluene remain highly correlated. Therefore we fixed $F$ at a $5.327 \mathrm{~cm}^{-1}$ value that corresponds to $I_{\alpha} \approx$ $3.2 \mu \AA^{2}$. The high correlation in both cases says that the confidence intervals obtained for the $V_{6}$ barrier heights should be taken with caution and to our opinion the $\pm 1 \mathrm{~cm}^{-1}$ interval will be a more reasonable estimate of the barrier height determination uncertainty. In this sense the room temperature experiment which will give an opportunity to access $m=3$, -3 states would be very valuable, since the splitting between 
these two states gives a direct measure of $V_{6} \cdot{ }^{8}$ Also it should be noted that it was not possible with the current dataset to determine the sign of the $V_{6}$ potential term (see ref. 8 and 30 for a detailed discussion) and therefore we adopted the sign choice which was found to be appropriate for the parent toluene molecule (i.e. plus sign, see Table 1). Finally we would like to point out that the barrier heights obtained with the RAM36 program of $V_{6}=0.14872(24)$ and $0.0856(10) \mathrm{kJ} \mathrm{mol}^{-1}$ for 2,6- and 3,5-difluorotoluene, respectively, are of the same order of magnitude as determined for the toluene. Conversely, the XIAM code, which was not written for $V_{6}$-symmetry, gives more than one order of magnitude higher barrier.

Once the transitions due to the parent species were identified and analyzed, it was straightforward to locate the lines due to the monosubstituted ${ }^{13} \mathrm{C}$ isotopologues for all independent ring positions and the methyl group. All isotopic lines were measured at their natural abundance ( $c a .1 \%$ ), so the number of lines measured for the monosubstituted ${ }^{13} \mathrm{C}$ isotopologues was rather sparse. Since we were mainly interested in the rotational constants the following approach to the data analysis was adopted. The torsional parameters $F$ and $V_{6}$ as well as quartic centrifugal distortion parameters were fixed at the parent species values and only $A, B, C$ and $\rho$ were varied together with some appropriate higher order terms. The derived rotational constants of the isotopic species are given in Tables S1 and S2 (ESI $\dagger$ ). The assigned transition frequencies together with the residuals of all the fits are given in the ESI† (Tables S5-S16).

There is one additional issue that should be discussed in connection with the monosubstituted ${ }^{13} \mathrm{C}$ isotopologues of 2,6and 3,5-difluorotoluene. The ${ }^{13} \mathrm{C}$ atoms substituted at positions (4), (1), (7) along the $C_{2}$ axis do not affect the $G_{12}$ symmetry of the system. Therefore for these isotopologues the $V_{6}$ treatment described above is exact. At the same time ${ }^{13} \mathrm{C}$ atoms substituted off-axis in the $(3)=(5)$ or $(2)=(6)$ positions destroy the $G_{12}$ symmetry of the problem and the $V_{3}$ treatment with a $G_{6}$ group should be applied. This is clearly seen from a comparison of relative intensities of ${ }^{\mathrm{b}} R_{ \pm 1,1}$ transitions with $K_{\mathrm{a}}=0,1$ which form close pairs of lines in the spectrum of 2,6-difluorotoluene. The relative intensities of these transitions are governed by the nuclear spin statistic weights which assume the 3 to 5 ratio in the case of $G_{12}$ symmetry and the 1 to 1 ratio when $G_{12}$ symmetry is destroyed and the $G_{6}$ group should be applied. This is what indeed was observed in our spectral records for 2,6-difluorotoluene. Therefore the $V_{3}$ mode of the RAM36 program ${ }^{8}$ was used for nonsymmetrical substituted isotopologues of 2,6- and 3,5difluorotoluene. It should be noted that in this case the axis system in use does not coincide with the principal axis system of the molecule. Since the isotopic perturbation is assumed to be rather small it is expected that the $V_{6}$ term in a Fourier expansion of the potential would be of the same order, but a small or comparable $V_{3}$ term will appear. Nevertheless our analysis shows that $(3)=(5)$ and $(2)=(6){ }^{13} \mathrm{C}$ isotopologues are fitted without any problem using the $V_{6}$ model and therefore there is no information available in the experimental data for the ratio between the $V_{3} / V_{6}$ terms (the attempts to introduce the $V_{3}$ term in the model lead to divergence of the fits). So the issue of the $V_{3} / V_{6}$ ratio in the case of small deviation from the
$G_{12}$ symmetry appeared to be beyond the scope of the current investigation though we note in passing that it is possible that the $V_{3}$ term in this case is much lower than the $V_{6}$ term. The results of the structural analysis based on these data are discussed in Section 3.3.

\subsection{Electric dipole moment}

The electric dipole moment components of both molecules were evaluated using the Stark effect. For this analysis it is desirable to limit the measurements to low $J$ transitions, which involve a smaller number of spatial $\left(M_{J}\right)$ components. Transitions involving low- $J$ values are also especially valuable when using coaxially aligned electrodes for Stark-effect applied in resonators (CAESAR), since the selection rule $\Delta M_{J}= \pm 1$ gives rise to a higher (approximately twice) number of $M_{J}$ components compared to the common parallel plate arrangement where $\Delta M_{J}=0$ transitions are observed. In order to determine the dipole moment we selected the set of rotational transitions and electric fields given in the ESI $\dagger$ (Tables S17 and S18). Fig. 3 illustrates the Stark effect measurements, showing transitions of the two title compounds. A fit of several $A$ species transitions, each of them measured at different $E$-fields, was done with the program QSTARK developed by Kisiel, ${ }^{31}$ and yielded dipole moment components of $\left|\mu_{\mathrm{b}}\right|=0.907(2) \mathrm{D}$ for 2,6-difluorotoluene and $\left|\mu_{\mathrm{b}}\right|=2.059$ (2) D for 3,5-difluorotoluene. These values are compared with the predictions in Table 1.

\subsection{Molecular structure}

The molecular structures of the title compounds were derived from the multiple isotopic data. The position of each substituted carbon atom was first calculated using the substitution method $\left(r_{\mathrm{s}}\right)$ of Kraitchman, which provides the absolute atomic coordinates in the principal axis system. ${ }^{32}$ This method computes the position of each atom without assumptions about the rest of the molecule, only from the changes in the moments of inertia upon isotopic substitution. This approach exploits the differences in the moments of inertia of different isotopic species rather than their absolute values achieving a partial cancellation of the inherent vibrational contributions contained in the ground-state moments of inertia. This procedure is somewhat affected by slightly different contributions to the moments of inertia of different isotopologues that become noticeable for atoms close to one of the inertial axes. ${ }^{33}$ Three carbon atoms (C1, C4 and C7) are lying along the $b\left(\equiv C_{2}\right)$ inertial axis in 2,6- and 3,5-difluorotoluene. Additionally, the a coordinates of atoms C2 and C6 are very close to the a axis in 2,6-difluorotoluene. For this reason, their sets of coordinates exhibited imaginary values of the atomic positions and had to be fixed to zero, hampering the accuracy of the structural determination, as in other high-symmetry molecules. The resulting atomic coordinates for the two molecules are compared with the $a b$ initio data in the ESI $\dagger$ (Tables S3 and S4). The derived bond lengths and valence angles are collected in Table 2. However, this procedure is unreliable for atoms close to one of the inertial axes ${ }^{34}$ and the results were unrealistic. For this reason we tried an effective structure $\left(r_{0}\right)$ calculation, in which the rotational constants were fitted to a selection of structural parameters. The derived bond lengths and valence angles assuming 


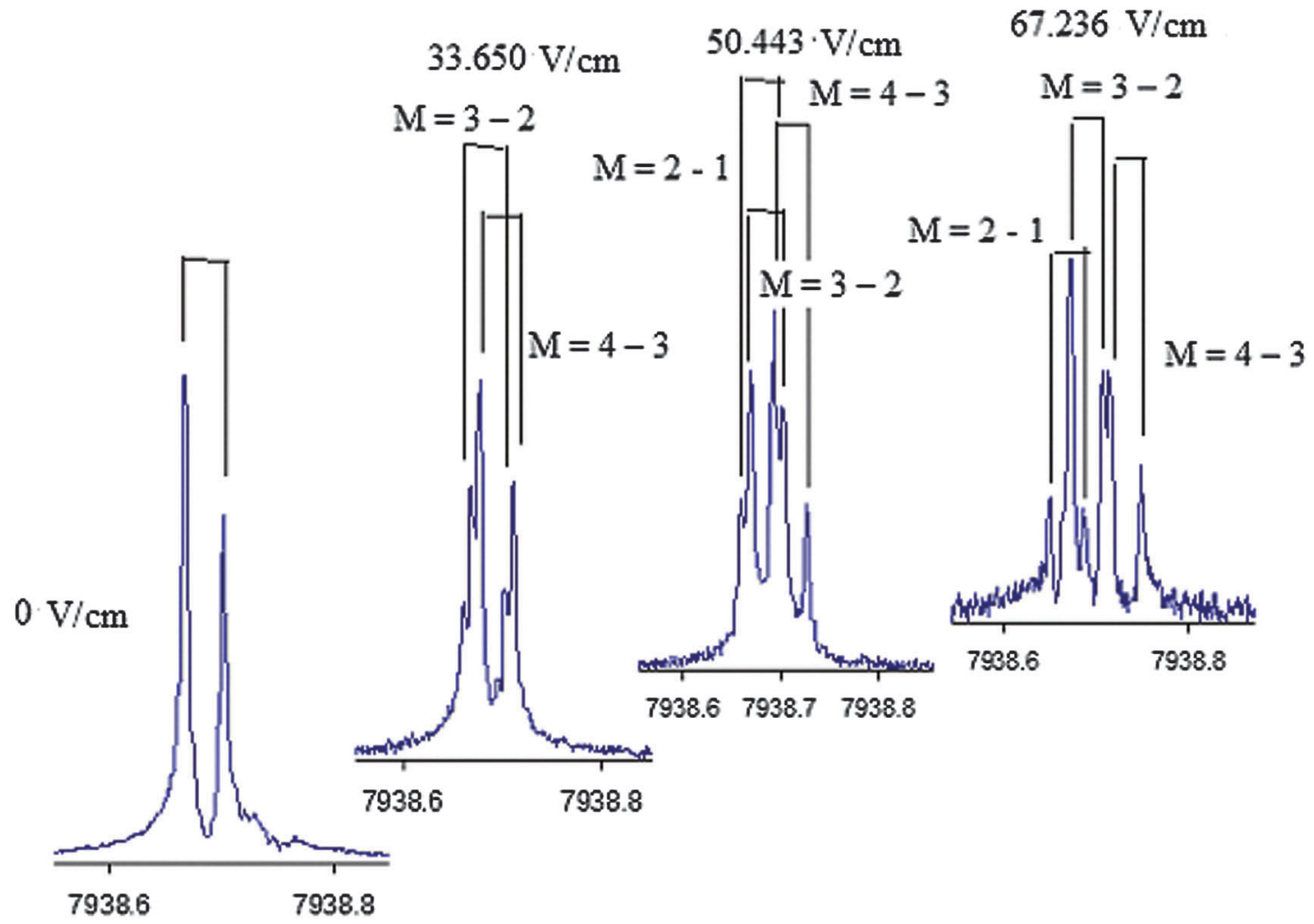

Fig. 3 Illustration of Stark effect measurements on the $J_{K-1, k+1}=4_{1,4} \leftarrow 3_{0,3}$ rotational transition of 3,5-difluorotoluene.

Table 2 Molecular structure of 2,6- and 3,5-difluorotoluene

\begin{tabular}{|c|c|c|c|c|c|c|}
\hline & \multicolumn{3}{|c|}{$\underline{\text { 2,6-Difluorotoluene }}$} & \multicolumn{3}{|c|}{ 3,5-Difluorotoluene } \\
\hline & $r_{\mathrm{s}}$ & $r_{0}$ & $r_{\mathrm{e}}^{c}$ & $r_{\mathrm{s}}$ & $r_{0}$ & $r_{\mathrm{e}}^{c}$ \\
\hline$r^{a}(\mathrm{C} 1-\mathrm{C} 2)$ & $1.36899(8)$ & $1.387(3)$ & 1.391 & $1.40483(5)$ & $1.403(2)$ & 1.395 \\
\hline$r(\mathrm{C} 3-\mathrm{C} 4)$ & $1.39371(5)$ & $1.400(5)$ & 1.391 & $1.38534(6)$ & $1.405(12)$ & 1.384 \\
\hline$r(\mathrm{C} 1-\mathrm{C} 7)$ & $1.50635(2)$ & $1.515(4)$ & 1.497 & $1.51174(4)$ & $1.509(18)$ & 1.502 \\
\hline$r(\mathrm{C}-\mathrm{F})$ & & $1.358(5)$ & 1.346 & & $1.354(9)$ & 1.344 \\
\hline$\angle(\mathrm{C} 3-\mathrm{C} 4-\mathrm{C} 5)$ & $120.265(8)$ & $120.5(1)^{d}$ & 120.4 & $115.602(7)$ & $115.1(6)^{d}$ & 116.5 \\
\hline$\angle(\mathrm{C} 2-\mathrm{C} 1-\mathrm{C} 6)$ & $117.29(1)$ & $115.1(3)^{d}$ & 115.1 & 118.993(6) & $119.3(2)^{d}$ & 120.4 \\
\hline$\angle(\mathrm{C} 2-\mathrm{C} 1-\mathrm{C} 7)$ & $121.354(7)$ & {$[122.5]^{e}$} & 122.5 & $120.504(7)$ & {$[120.4]^{e}$} & 120.4 \\
\hline$\angle(\mathrm{C}-\mathrm{C}-\mathrm{F})$ & & $117.5(3)$ & 117.8 & & $120.3(10)$ & 118.9 \\
\hline
\end{tabular}

only a planar heavy atom skeleton and a symmetric $\mathrm{C} 7$ position are also collected in Table 2, together with the theoretical nearequilibrium $\left(r_{\mathrm{e}}\right)$ ab initio values.

\section{Discussion and conclusions}

We have observed the rotational spectra of two symmetrically disubstituted fluorine derivatives of toluene in positions ortho and meta. The spectrum is dominated by the large torsional doublings arising from the pure six-fold potential barriers hindering internal rotation. In order to simplify the spectrum analysis the experiment was conducted in a supersonic jet expansion, allowing the detection of the lowest-lying torsional substates of the ground vibrational state. Stark effect measurements yielded the electric dipole moments, which confirm the larger polarity of 3,5-difluorotoluene $(2.00 \mathrm{D} v s .0 .88 \mathrm{D})$, and only differ by $3 \%$ to the ab initio calculations. The sensitivity of our experiment allowed the additional observation of all ${ }^{13} \mathrm{C}$ monosubstituted isotopologues in natural abundance. Structural calculations using the substitution and effective methods provided accurate information on the carbon skeleton of the molecule. The main objectives of this work, including the rotational assignment of the title compounds, the determination of molecular structures, the calculation of the electric dipole moments and the comparison of the internal rotation potential barriers with that of toluene, 
Table 3 Internal rotation barriers in toluene derivatives

\begin{tabular}{|c|c|c|}
\hline Molecule & $V_{6}\left(\mathrm{~J} \mathrm{~mol}{ }^{-1}\right)$ & $\mu(\mathrm{D})$ \\
\hline Toluene $^{a}$ & $57.87439532(13)$ & 0.37 \\
\hline$p$-Fluorotoluene $\mathrm{e}^{b, c, d}$ & $57.777(76)$ & $1.96(2)$ \\
\hline$p$-Chlorotoluene $e^{e}$ & $58.28(17)$ & $1.71(2)$ \\
\hline 3,5 -Difluorotoluene ${ }^{f}$ & $85.6(10)$ & $2.059(2)$ \\
\hline 2,6-Difluorotoluene ${ }^{f}$ & $148.72(24)$ & $0.907(2)$ \\
\hline
\end{tabular}

are thus fully accomplished, both experimentally and theoretically. In particular, the difficult internal rotation problem was solved thanks to a new computer program specially designed for six-fold $V_{6}$ problems. $^{8}$ Comparisons are now possible between the experimental and theoretical predictions for the most relevant molecular properties of 2,6- and 3,5-difluorotoluene in Table 1 . The $a b$ initio predictions show a reasonable description of the rotational parameters (relative deviations below $c a .0 .8 \%$ ). However, ab initio predictions of the internal rotation barrier are much more difficult, not unexpected considering the very small magnitude of the potential barriers. The theoretical predictions suggest that the barrier in 3,5-difluorotoluene is more than one order of magnitude larger than in 2,6-difluorotoluene (0.16 and $0.006 \mathrm{~kJ} \mathrm{~mol}^{-1}$, respectively), failing to reproduce the magnitude and reversed trend of the experimental values in 2,6- $\left(0.148 \mathrm{~kJ} \mathrm{~mol}^{-1}\right)$ vs. the more polar 3,5-difluorotoluene $\left(0.0856 \mathrm{~kJ} \mathrm{~mol}^{-1}\right)$. In conclusion, the computationally effective MP2 method proves effective for the prediction of spectral properties, but a more accurate description of the small torsional barriers seems to require a more advanced calculation, which is out of the scope of the present work. Comparisons of the barrier to internal rotation in toluene and mono and disubstituted halotoluenes is also revealing. A comparison of available experimental data in Table 3 shows that para-monosubstituted fluoro or chlorotoluenes have barriers very similar to toluene (0.0578 and 0.0583 vs. $0.05787 \mathrm{~kJ} \mathrm{~mol}^{-1}$, respectively), suggesting that there is a small effect of the substituents in the para position on the bonding characteristics of $\mathrm{C}-\mathrm{CH}_{3}$ of toluene. Conversely, the introduction of two symmetric electronegative substituents in ortho or meta positions considerably increases the internal rotation barrier $\left(0.085\right.$ and $0.148 \mathrm{~kJ} \mathrm{~mol}^{-1}$, respectively, in meta and ortho) reflecting a much larger influence in the molecular orbital distribution of the halotoluenes.

In conclusion, the investigation of internal rotation barriers in toluenes with different halogenated substituents will progressively provide a general view of the subtle effects affecting intramolecular dynamics in these molecules. To this effect the combination of experimental data, new spectroscopic methods and $a b$ initio calculations proves to be extremely efficient.

\section{Acknowledgements}

We thank the Land Niedersachsen and the Deutsche Forschungsgemeinschaft (DFG) for financial support. A. L. acknowledges the financial support from the Spanish MINECO (CTQ201239132-C02-02).

\section{References}

1 D. G. Lister, J. N. MacDonald and N. L. Owen, Internal Rotation and Inversion: An Introduction to Large Amplitude Motions in Molecules, Academic Press, New York, 1978.

2 J.-U. Grabow and W. Caminati, in Frontiers of Molecular Spectroscopy, ed. J. Laane, Elsevier, Amsterdam, 2009, ch. 14.

3 W. Caminati and J.-U. Grabow, in Frontiers of Molecular Spectroscopy, ed. J. Laane, Elsevier, Amsterdam, 2009, ch. 15.

4 W. Gordy and R. L. Cook, Microwave Molecular Spectra, Wiley, New York, 1984.

5 I. Kleiner, J. Mol. Spectrosc., 2010, 260, 1.

6 H. D. Rudolph, H. Dreizler, A. Jaeschke and P. Wendling, Zeitschrift für Naturforschung A, 1970, 22, 940-944.

7 Z. Kisiel, E. Bialkowska-Jaworska, L. Pszczółkowski and H. Maeder, J. Mol. Spectrosc., 2004, 227, 109-113.

8 V. V. Ilyushin, Z. Kisiel, L. Pszczólkowski, H. Mäder and J. T. Hougen, J. Mol. Spectrosc., 2010, 259, 26.

9 W. A. Kreiner, H. D. Rudolph and B. T. Tan, J. Mol. Spectrosc., 1973, 48, 86-99.

10 V. Amir-Ebrahimi, A. Choplin, J. Demaison and G. Roussy, J. Mol. Spectrosc., 1981, 89, 42-52.

11 J. R. Gascooke, E. A. Virgo and W. D. Lawrance, J. Chem. Phys., 2015, 142, 024315.

12 J. R. Gascooke, E. A. Virgo and W. D. Lawrance, J. Chem. Phys., 2015, 143, 044313.

13 R. A. Walker, E. C. Richard, K. T. Lu and J. C. Weisshaar, J. Phys. Chem., 1995, 99, 12422.

14 (a) H. D. Rudolph, H. Dreizler, A. Jaeschke and P. Wendling, Zeitschrift für Naturforschung A, 1967, 22, 940-944; (b) J. Susskind, J. Chem. Phys., 1970, 53, 2492-2501; (c) D. Schwoch and H. D. Rudolph, J. Mol. Spectrosc., 1975, 57, 47-74; (d) S. Jacobsen, U. Andersen and H. Maeder, Struct. Chem., 2003, 14, 217.

15 H. D. Rudolph and A. Trinkhaus, Zeitschrift für Naturforschung A, 1968, 23, 68-76.

16 (a) H. D. Rudolph and H. Seiler, Zeitschrift für Naturforschung A, 1965, 20, 1682-1686; (b) J. Rottstegge, H. Hartwig and H. Dreizler, J. Mol. Struct., 1999, 478, 37-47; (c) G. E. Heberich, Zeitschrift für Naturforschung A, 1967, 22, 761-764; (d) P. N. Ghosh, J. Mol. Spectrosc., 1989, 138, 505.

17 (a) K. P. R. Nair and K. Epple, Chem. Phys. Lett., 1990, 166, 146-152; (b) K. P. R. Nair, J. Mol. Struct., 1999, 477, 251-254; (c) K. P. R. Nair and S. Eappen, Indian J. Pure Appl. Phys., 2001, 39, 750-751; (d) D. Gerhard, A. Hellweg, I. Merke, W. Stahl, M. Baudelet, M. Petitprez and G. Wlodarczak, J. Mol. Spectrosc., 2003, 220, 234-241; (e) K. P. R. Nair, J. Demaison, G. Wlodarczak and I. Merke, J. Mol. Spectrosc., 2006, 237, 137-142.

18 H. D. Rudolph, K. Walzerm and I. Krutzik, J. Mol. Spectrosc., 1973, 47, 314-339.

19 S. Maiti, A. I. Jaman and R. N. Nandi, J. Mol. Spectrosc., 1996, 177, 29-33.

20 (a) J.-U. Grabow and W. Stahl, Zeitschrift für Naturforschung A, 1990, 45, 1043-1044; (b) J.-U. Grabow, W. Stahl and H. Dreizler, Rev. Sci. Instrum., 1996, 67, 4072-4084. 
21 A. Lesarri, J.-U. Grabow, Asian J. Spectrosc., 2010, special issue, 1-14.

22 J.-U. Grabow, Habilitationschrift, Hannover University, 1994.

23 M. Schnell, D. Banser and J.-U. Grabow, Rev. Sci. Instrum., 2004, 75, 2111.

24 J. M. L. J. Reinarts and A. Dymanus, Chem. Phys. Lett., 1974, 24, 346-351.

25 Z. Kisiel, J. Kosarzewski and B. A. Pietrewicz, Chem. Phys. Lett., 2000, 325, 523.

26 M. J. Frish, et al., GAUSSIAN03, Revision B 04, Gaussian Inc., Pittsburgh, PA, 2003.

27 P. R. Bunker and P. Jensen, Molecular Symmetry and Spectroscopy, NRC Research Press, Ottawa, 2nd edn, 1998, p. 511.

28 H. Hartwig and H. Dreizler, Zeitschrift für Naturforschung A, 1996, 51, 923.
29 G. O. Sorensen and T. Pedersen, Stud. Phys. Theor. Chem., 1983, 23, 219-236.

30 V. V. Ilyushin, L. B. Favero, W. Caminati and J.-U. Grabow, ChemPhysChem, 2010, 11, 2589-2593.

31 Z. Kisiel, B. A. Pietrewicz and F. W. Fowler, J. Phys. Chem. A, 2000, 104, 6970.

32 Equilibrium Molecular Structures, ed. J. Demaison, J. E. Boggs and A. G. Császár, CRC Press, Boca Raton, 2011.

33 J. Demaison and H. D. Rudolph, J. Mol. Spectrosc., 2002, 215, 78.

34 H. D. Rudolph, Determination of the Structural Parameters from the Inertial Moments, in Equilibrium Molecular Structures: From Spectroscopy to Quantum Chemistry, ed. J. Demaison, J. E. Boggs and A. G. Csaszar, CRC Press, Boca Ratón, FL, 2010. 